\title{
L'IMPATTO DEL VIRUS CORONA-19 SUL DIRITTO DELLA CRISI
}

\author{
THE IMPACT OF THE CORONA-19 VIRUS ON THE LAW OF THE CRISIS \\ O IMPACTO DO VÍRUS CORONA-19 NA LEI DA CRISE
}

\author{
Stefania Pacchi \\ Dottorato di ricerca a L'Università di Siena \\ Università di Siena \\ 1’Università La Sapienza di Roma \\ Università Carlos III di Madrid \\ stefania.pacchi@gmail.com
}

\begin{abstract}
Riassunto: A causa dell'esplosione del Covid-19 e del conseguente blocco, terribili problemi hanno colpito le aziende. Il governo italiano, come gli altri, si è adoperato per limitare i danni e scongiurare lo spettro di tanti fallimenti immediati. Quattro decreti legge (denominati Cura Italia, Liquidità, Rilancio, Semplificazione), adottati lungo una linea relativa all'andamento della situazione sanitaria, hanno introdotto misure con impatto diretto sulle aziende e sulle opzioni gestionali del amministratori. Gli obiettivi di questi quattro interventi differiscono: mentre il primo è una misura di contenimento dei danni, il secondo è sostenere la crisi di liquidità, il terzo per la riattivazione del sistema imprenditoriale e il quarto per favorire gli investimenti pubblici e la ripresa delle attività. l'economia. L'articolo illustra i principali interventi per aiutare le aziende colpite dal blocco.
\end{abstract}

Parole chiave: Crisi d'impresa. Insolvenza. Crisi da Covid-19.

Abstract: Due to the explosion of Covid-19 and the consequent lock-down, terrible problems have affected companies. The italian Government, like the others, has taken steps to limit the damages and avoid the specter of many immediate bankruptcies. There are four decree-laws (called Cura Italia, Liquidità, Rilancio, Semplificazione), adopted along a line related to the progress of the health situation, have introduced measures with a direct impact on companies and on the management options of the administrators. The objectives of these four interventions differ: while the first is a damage containment measure, the second aims to support the liquidity crisis, the third for the reactivation of the business system and the fourth to encourage public investment and the restart of the economy. The article illustrates the main interventions to help companies affected by the lockdown.

Keywords: Business crisis. Insolvency. Covid-19 crisis.

Resumo: Devido à pandemia da Covid-19 e o consequente lockdown, problemas terríveis afetaram as empresas. O Governo italiano, como os outros, tomou medidas para limitar os danos e evitar o espectro de muitas falências imediatas. Quatro decretos-lei (denominados Cura Italia, Liquidità, Rilancio, Semplificazione), adotados ao longo de uma linha relacionada com a evolução da situação da saúde, introduziram medidas com impacto direto nas empresas e nas opções de gestão dos administradores. Os objetivos destas quatro intervenções são diferentes: enquanto a primeira é uma medida de contenção de danos, a segunda objetiva apoiar a crise de liquidez, a terceira a reativação do sistema empresarial e a quarta visa incentivar o investimento público e o reinicio da economia. $\mathrm{O}$ artigo ilustra as principais intervenções para ajudar as empresas afetadas pelo lockdown.

Palavras-chave: Crise empresarial. Insolvência. Crise da Covid-19. 


\section{Para citar este artigo}

ABNT NBR 6023:2018

PACCHI, Stefania. L'impatto del virus Corona-19 sul Diritto Della Crisi. Prisma Jurídico, São Paulo, v. 19, n. 2, p. 319-346, jul./dez. 2020. http://doi.org/10.5585/prismaj.v19n2.18706.

\section{Premessa}

Se John Kenneth Galbraith si trovasse a vivere la stagione attuale probabilmente non saprebbe quale titolo dare a una sua pubblicazione che volesse illustrarla, atteso che l'incertezza che oggi contrassegna l'economia è ben superiore a quella che lo Scrittore individuava, a fine anni '70, nell'età della crisi petrolifera.

Non vi è dubbio che gli effetti della pandemia, difficilmente enucleabili e quantificabili, generano incertezza rendendo estremamente aleatorio il compito di chi, giurista o aziendalista, sia chiamato a dare risposte agli interrogativi sul futuro delle imprese. Sono risposte tanto più difficili quanto più gli eventi spingono verso decisioni rapide. L'impresa, che non ha tollerato le soluzioni di continuità verificatesi negli ultimi mesi, esige ora interventi urgenti perché si sta configurando, grazie all'interconnessione dei mercati, una situazione di pericolo estremo, per tutte le economie e la collettività causato dalla massa di debiti immessa sul mercato per cercare di alleviare gli effetti economici della crisi. Secondo un rapporto dell'Institute of International Finance (IIF), insieme a questo aumento dell'indebitamento, le inadempienze stanno già raggiungendo record in termini assoluti.

Ciò ha fin da subito reso evidente che le conseguenze e gli effetti che il COVID-19 produce in tutto il mondo portano a un'enorme crisi di liquidità, in particolare per le micro, le piccole e medie imprese. Il Fondo monetario internazionale ha avvertito che il tasso di fallimento per le piccole e le medie imprese potrebbe triplicare quest'anno in assenza di un sostegno statale sufficiente, minacciando di bloccare la ripresa economica e causare instabilità finanziaria. Sulla base di un'analisi condotta in 17 Paesi, il FMI prevede che i fallimenti potrebbero salire al $12 \%$, dal $4 \%$ prima della pandemia. L'Italia vedrebbe il maggiore aumento a causa di un forte calo della domanda aggregata e dell'elevata quota di produzione nelle industrie ad alta intensità di contatto.

Anche se il rapporto tra crisi economica e numero dei fallimenti non è sempre direttamente proporzionale, è certo che la situazione di grave carenza di liquidità indotta dalle misure di contenimento del virus Corona-19 e non superata, potrà comportare un aumento delle procedure liquidative superiore a quello provocato da altre recessioni. 
La conseguenza di un numero alto di fallimenti, però, impatterà fortemente non solo sull'economia ma anche sul funzionamento dei tribunali. Tutto ciò è preoccupante soprattutto perché, come risulta da uno studio condotto dalla Brigham Young University, le piccole e medie imprese hanno ancora più probabilità di essere liquidate in un tribunale congestionato da una mole di procedure che in un tribunale con un carico gestibile. Inoltre, sebbene le implicazioni non siano così disastrose per le grandi società, tuttavia anche per queste, se il tribunale è congestionato, ci vuole più tempo per riorganizzarle e i costi sono sostanzialmente più elevati. Ciò perché i giudici sono chiamati a prendere decisioni critiche, in condizioni di estrema pressione, sulla base di informazioni di parte, quindi altamente contestate e generatrici di conflitti.

Queste criticità del sistema giudiziario significano che la legge fallimentare non può servire come risposta politica principale alle tensioni che oggi le imprese stanno affrontando.

Da una parte la procedura esecutiva o concorsuale non fornisce al consumatore i rimedi essenziali di cui ha bisogno in questo momento: liquidità e tolleranza. Dall'altra, le piccole imprese potrebbero forse attingere liquidità in alcune procedure vista la protezione che i finanziatori trovano, ma il diluvio di casi travolgerebbe i tribunali fallimentari.

Per la piccola impresa prototipica più che predisporre procedure concorsuali occorre assicurare la presenza del proprietario manager che però deve essere incentivato. Per queste imprese è fondamentale la sospensione della regola della priorità assoluta. In Italia, a tal fine, è venuto in soccorso l'art. 8 d.1. 23/2020 che parifica il finanziatore agli altri creditori disponendo che, benché la società sia in una situazione di indebitamente eccessivo rispetto al patrimonio netto, in cui sarebbe ragionevole un conferimento a capitale, il finanziamento effettuato dal socio non determina la sua postergazione.

\section{Le urgenze: scongiurare la dichiarazione di fallimento}

È emersa, quindi, nella prima fase di gestione degli effetti del lock-down la necessità di allontanare nel tempo la sottoposizione alla procedura concorsuale liquidatoria.

L'art. 10 del d.1. 23 del 2020 (conv. nella L.40/2020) dispone che i ricorsi per la dichiarazione di fallimento e per la dichiarazione di insolvenza (funzionale all'instaurazione delle procedure di liquidazione coatta amministrativa e di amministrazione straordinaria delle grandi imprese insolventi) presentati tra il 9 marzo 2020 ed il 30 giugno 2020 sono improcedibili. Rimangono fuori dalla previsione gli istituti del sovraindebitamento, "probabilmente essendo stato ritenuto sufficiente il pacchetto di blocco delle vendite coattive, 
la più generale sospensione dei termini processuali, nonché le dilatate scadenze dei titoli di credito (art. 11 d.1. n. 23 del 2020)".

Se prima della conversione in legge del d.1. 23/2020 si poteva ritenere che, poiché letteralmente l'improcedibilità si riferiva ai "ricorsi ai sensi degli articoli 15 e 195 del r.d. 16 marzo 1942, n. 267", vista la ratio della disposizione, si estendesse anche ai casi di conversione della procedura di concordato preventivo in fallimento per revoca ex art. 173 1. fall., di diniego di ammissione ex art. 162 e di omologazione ex art. 180 1.fall., adesso sulla base del comma 2 lett.b) della legge di conversione 40/2020, queste ipotesi devono essere escluse ogni volta che vi sia istanza di uno dei soggetti legittimati a presentarla. Ritiene, quindi il legislatore - e a mio avviso correttamente - che dinanzi a fattispecie concordatarie già oggetto di valutazione negativa si debba procedere alla dichiarazione di fallimento.

Ulteriori casi costituiscono eccezioni alla improcedibilità. Innanzi tutto, quando il fallimento (o la dichiarazione di insolvenza) è chiesto dal debitore in proprio, se però l'insolvenza non è conseguenza dell'epidemia di Covid-19, con ciò confermando che gli è imposta, invece, una pausa di riflessione quando così non sia.

In secondo luogo, sfugge all'improcedibilità l'istanza del PM quando contestualmente siano richiesti provvedimenti cautelari o conservativi a tutela del patrimonio o dell'impresa (art. 15 , comma 8,1 . fall.) o quando la richiesta è presentata ai sensi dell'art. 7 , numero 1 , non potendo comportamenti anomali o atti di frode sfuggire all'accertamento penale grazie a un rinvio della dichiarazione di fallimento.

Il tribunale non darà corso all'istruttoria prefallimentare e, quindi, - a parte le fattispecie espressamente escluse - qualunque impresa, sia essa "affetta dal virus" o invece sia già in crisi ex ante, è stata sottratta fino al 30 giugno 2020 alla procedura. La soluzione legislativa in primo luogo vuole evidentemente evitare nella prima fase di gestione della pandemia una verifica del requisito circa il momento di insorgenza della crisi che avrebbe notevolmente appesantito il lavoro dei tribunali. In secondo luogo, mira ad offrire a tutte le imprese indistintamente un momento di tregua probabilmente ritenendo che il danno per il ritardo della sottoposizione a fallimento delle imprese già in crisi fosse limitato proprio in ragione del lock-down. È chiaro però che togliere ai creditori lo strumento dell'istanza di fallimento del debitore costituisce, proprio per la crisi generalizzata, un rimedio pericoloso.

Di conseguenza il legislatore cerca di bilanciare queste posizioni prevedendo nel comma 3 dell'art. 10 della L. 40/2020 che, se intervenga entro il 30 settembre 2020 la dichiarazione di fallimento collegata alle istanze dichiarate improcedibili fino al 30 giugno, non viene computato quel periodo di “congelamento” ai fini dell'esercizio dell'azione revocatoria. 
Sempre nell'ottica di mettere sotto la campana di vetro l'impresa - dopo avere inertizzato i ricorsi per il fallimento - è intervenuto l'art. 6 del d.1. 2 /2020 prevedendo che dal 9 aprile 2020 fino al 31 dicembre 2020 non si applicano gli articoli 2446, commi 2 e 3, 2447, 2482-bis, commi 4, 5 e 6, e 2482-ter del codice civile. Per lo stesso periodo non opera la causa di scioglimento della società per riduzione o perdita del capitale sociale di cui agli articoli 2484, comma 1, numero 4), e 2545-duodecies del codice civile. la ratio è di liberare la società (i suoi soci e gli amministratori) dall'alternativa tra l'incudine (ricapitalizzare) e il martello (liquidare) per una situazione generale dipendente dall'emergenza COVID-19.

Non viene meno, tuttavia, l'obbligo degli amministratori di convocare senza indugio l'assemblea per informare i soci e l'adozione dei provvedimenti opportuni.

Naturalmente, essendo disapplicata la causa di scioglimento viene meno la responsabilità degli amministratori $e x$ art. 2486 c.c. (dovere di gestione conservativa). Tuttavia, la causa di scioglimento è disapplicata solo dal 9 aprile 2020. Pertanto, se la causa di scioglimento si fosse verificata prima di tale data, per il periodo anteriore resta ferma la responsabilità degli amministratori (cfr. ancora l'art. 182-sexies 1. fall.).

L'intervento emergenziale ha congelato, quindi, la situazione per l'impresa e per i suoi creditori nella convinzione che allo stato la liquidazione fallimentare, con la difficoltà estrema di vendere i beni, può soltanto determinare un'atomizzazione del complesso produttivo con perdita di ogni valore senza peraltro poter recare vantaggio alcuno ai creditori.

È importante, infatti, riconoscere il rischio di "rapinare Peter per pagare Paul" che oggi si corre in caso di fallimento. 11 Covid-19 "infetta" qualsiasi procedura, perfino il fallimento che potrebbe apparire "refrattario" al terribile virus.

Questa procedura, che può essere lo strumento per conservare i valori attraverso una cessione unitaria realizzata al suo interno o in un concordato fallimentare, oggi appare soltanto funzionale alla disgregazione. Pare illusorio pensare che in un mercato di imprese affette da difficoltà generalizzate si possano intercettare imprenditori disposti a rilevare complessi aziendali o anche soltanto immobili o blocchi di beni. Parimenti remota è l'ipotesi sia di un esercizio dell'impresa da parte del curatore con l'obbiettivo di conservare in attività l'azienda in attesa di un compratore, sia quella di un affitto d'azienda realizzato in procedura al fine di traghettare l'impresa in altre mani. La possibile funzione conservativa del fallimento, quindi, viene di fatto tarpata dal COVID-19. 


\section{Le urgenze: mettere al riparo le procedure compositive in corso}

Come successivo passo, il Legislatore ha cercato di evitare il naufragio delle procedure preventive in corso. La continuità è stata assunta come obbiettivo. A tale scopo il d.l. 8 aprile 2020, n. 23 (decreto Liquidità, conv. nella L. 40/2020) ha introdotto alcune "Misure urgenti per garantire la continuità delle imprese colpite dall'emergenza Covid-19" consistenti, in primis, in deroghe di carattere transitorio ad alcune disposizioni in materia di diritto societario e fallimentare, che in parte ricalcano iniziative adottate da Legislatori di altri Paesi. Per quanto riguarda le procedure in corso si è avuta, grazie all'art. 9 del d.1.23/2020, una sorta di rimodulazione dei concordati e degli accordi di ristrutturazione per evitare il naufragio di procedure in corso e caratterizzate da fondate aspettative di esito positivo. In sostanza si tratta di una serie di misure atte a favorire l'adempimento dei concordati (e degli accordi di ristrutturazione).

Innanzi tutto, sono stati prorogati di sei mesi i termini di adempimento dei concordati preventivi e degli accordi di ristrutturazione omologati aventi scadenza successiva al 23 febbraio 2020.

Per queste procedure non è tuttavia prevista la possibilità di rinegoziare il contenuto della proposta o dell'accordo che avrebbe altrimenti condotto ad un imbiancamento di fatto di tutte le fasi già espletate.

La disposizione è evidentemente diretta ai concordati in continuità nei quali il soddisfacimento dei creditori è legato ai risultati dell'attività che, essendo stata bloccata, rischia di non poter far fronte agli impegni assunti. Per i concordati liquidativi l'impatto del Covid-19 si produce sulla vendita dei beni che si auspica possibile con una dilatazione dei tempi di esecuzione.

In secondo luogo, per i concordati preventivi e gli accordi di ristrutturazione non ancora omologati al 23 febbraio 2020, il debitore può presentare, sino all'udienza fissata per l'omologa, istanza al tribunale per la concessione di un termine non superiore a novanta giorni per il deposito di un nuovo piano e di una nuova proposta di concordato ai sensi dell'articolo 1611 . fall. o di un nuovo accordo di ristrutturazione ai sensi dell'articolo 182-bis 1. fall.

Il termine dei 90 giorni decorre dalla data del decreto con cui il Tribunale - senza valutazione alcuna sull'esistenza dei presupposti - lo assegna e non è prorogabile. L'istanza è inammissibile se presentata nell'ambito di un procedimento di concordato preventivo nel corso del quale è già stata tenuta l'adunanza dei creditori ma non sono state raggiunte le maggioranze stabilite dall'articolo 177 1. fall. 
Chiaramente la pendenza del processo di omologazione presuppone che i creditori avessero votato favorevolmente l'originaria proposta; se l'avessero già bocciata, questa norma non dà al debitore una seconda chance (in sostanza: il termine serve solo per ricalibrare un piano, proposta, accordo che già era stato approvato ma che ora in quei termini non è più economicamente fattibile). In quest'ultimo caso il debitore potrà, tuttavia, presentare un nuovo ricorso (corredato ab initio dalla proposta, dal piano e dai documenti), dal quale si desuma la rinuncia alla pregressa procedura. Ulteriore misura di favore è racchiusa poi nel comma 3 dell'art. 9, d.1. 23/2020 e consiste nella possibilità accordata al debitore di modificare i termini di adempimento del concordato preventivo o dell' accordo di ristrutturazione depositando, sino all'udienza fissata per l'omologa, una memoria contenente l'indicazione dei nuovi termini, insieme alla documentazione che comprovi la necessità della modifica dei termini. Il differimento dei termini non può essere superiore di sei mesi rispetto alle scadenze originarie. Il Tribunale non autorizza la modifica dei termini ma, riscontrata la sussistenza dei presupposti di cui agli articoli 180 o 182-bis 1. fall. e acquisito il parere del commissario giudiziale (il quale pare che debba esprimersi anche sulla modifica dei termini), procede all'omologa, dando espressamente atto delle nuove scadenze. È chiaro che con questa misura, grazie alla quale si accorda al debitore la possibilità di modificare soltanto i termini di adempimento, diversamente da quella disposta nel secondo comma - si prescinde dal consenso dei creditori, la tutela dei quali è riassunta nell'omologazione attraverso vuoi il controllo del giudice, vuoi l'esercizio del diritto di opposizione.

Procedendo nell'esame dell'art. 9, d.1. 23/2020, (quarto comma), in sede di concordato in bianco viene prevista la possibilità (anche nel caso in cui sia pendente una domanda di fallimento) di un'ulteriore ed eccezionale proroga di 90 giorni per la presentazione di piano, proposta e documentazione. Per il resto la disciplina di riferimento rimane quella racchiusa nei commi settimo e ottavo dell'art. 161 1.fall. La richiesta deve essere debitamente motivata con specifico riferimento ai fatti sopravvenuti per effetto dell'emergenza epidemiologica COVID19 e all'incidenza che quelli hanno avuto sull'attività generando problemi nella redazione del piano; su di essa il Tribunale raccoglie il parere del commissario giudiziale (se nominato) e decide sulla domanda. Lo stesso meccanismo si applica per la domanda di protezione anticipata ex art. 182-bis, comma 7, 1. fall.

Il tribunale, sulla base degli elementi e delle prove addotti dall'imprenditore, del parere del commissario, concederà la proroga se ravvisi l'effettiva utilità di una dilatazione del periodo di automatic stay sull'impresa, che sarà valutata in prospettiva così come le possibilità di invertire il trend che il lock-down ha impresso. Si tratta di una valutazione che dovrà avere come 
termine di riferimento l'interesse dei creditori, per cui il prolungamento dell'automatic-stay non potrebbe andare a svantaggio di loro. Sarà quindi una valutazione globale sugli strumenti (finanziamenti, nuovi investimenti, riorganizzazione dell'impresa, operazioni straordinarie ecc.) dei quali l'impresa potrà realisticamente usufruire per raggiungere l'obbiettivo del recupero dell'efficienza.

Se, sulla base dei diversi elementi caratterizzanti lo stato dell'impresa e le sue prospettive gestionali, il tribunale consegue un giudizio positivo sull'impresa, il tribunale accoglierà la richiesta del debitore di un ulteriore proroga. La disposizione esaminata viene replicata (quinto comma dell'art. 9) per gli accordi di ristrutturazione, ma in questa fattispecie il tribunale concederà il termine quando, esaminata la motivazione del debitore, possa valutare che sussistano ancora le condizioni per raggiungere il consenso della maggioranza richiesta dall'art. 182-bis.

\section{Le urgenze: la sequenza automatic stay - piano attestato}

Il Legislatore dopo aver disposto misure di ausilio alle imprese consistenti in un ampliamento dello spazio temporale per la negoziazione, o per l'esecuzione delle proposte inserite in concordati o in accordi e nella possibilità di rimodellare piano e proposta alla mutata situazione post-Covid, sfrutta lo schema e gli effetti del preconcordato scolpito nell'art. 161, sesto comma ((o del preaccordo di ristrutturazione ex art. 182-bis, settimo comma) per attribuire all'imprenditore uno spatium temporis coperto dalle aggressioni dei creditori e nel contempo sottoposto alla vigilanza del tribunale (con eventuale nomina di un precommissario) per redigere e presentare entro il termine concesso, un piano di risanamento attestato ai sensi dell'art. 67, terzo comma, lettera d), 1.fall.

La previsione è riservata al debitore che entro il 31 dicembre 2021 abbia ottenuto la concessione dei termini di cui all'art. 161, sesto comma, o all'art. 182-bis, settimo comma.

La sequenza dalla procedura concordataria o da quella finalizzata all'omologazione di un accordo di ristrutturazione al piano attestato passa dalla rinuncia alla procedura "in bianco" che porterà il giudice a verificare la completezza e la regolarità della documentazione inerente al piano per poi dichiarare l'improcedibilità del ricorso originariamente presentato e finalizzato all'ottenimento dei termini.

Con questa disposizione si innesta in una procedura giudiziale, quale quella di preaccordo o preconcordato, finalizzata ad ottenere la protezione delle trattative e, quindi, necessariamente munita dei presidi del controllo del tribunale sull'attività svolta e sulle 
operazioni compiute dal debitore in questa fase, uno strumento negoziale assolutamente stragiudiziale, ma pubblicato nel registro delle imprese, sfruttando la rinuncia al processo. In sostanza l'imprenditore, pur dichiarando fin dall'inizio che l'esito del periodo protetto potrebbe sfociare anche in un piano attestato, accetta una limitazione alla gestione e un controllo giudiziale in cambio di una protezione delle trattative e dell'approdo ad uno strumento meno invasivo, meno costoso e più duttile di quelli che altrimenti avrebbero costituito lo sbocco atteso.

Senza dubbio il piano attestato, che fugacemente appare nell'art. 67, terzo comma, lett. d) al limitato fine di essere inserito nell'alveo delle operazioni che possono produrre atti esenti da revocatoria ma che rimane comunque caratterizzato dall'assenza di controlli e "sigilli" giudiziali, si affaccia in tribunale sia pure per una verifica condotta esclusivamente sulla completezza e la regolarità della documentazione che giustificano il mantenimento degli effetti dal periodo protetto instaurato per consentire il consolidamento di una procedura giudiziale o semigiudiziale. La "storica" riservatezza del piano attestato viene meno ma questo è il prezzo che l'imprenditore paga per l'ausilio straordinario e temporalmente circoscritto. Il sistema perde in coerenza probabilmente scambiata per offrire uno strumento che potrebbe spingere le imprese a mettersi tempestivamente sotto il controllo del tribunale avendo dinanzi a sé come miraggio uno strumento comunque meno invasivo e non immediatamente contiguo al fallimento.

Ultima e importante disposizione è contenuta nell'art. 5-ter. Reiterando la volontà di attribuire all'imprenditore, colpito dal Covid-19, una pausa di sospensione per tentare di uscire dalle secche, la norma stabilisce che ai concordati depositati entro il 31 dicembre 2020 non si applica il disposto dell'art. 161, comma 10, e quindi non vi è il dimezzamento del termine per il deposito del piano nel caso in cui penda il procedimento per la dichiarazione di fallimento.

\section{Evitare il fallimento. si può rileggere l'art. 5 l.fall.?}

Mentre si allontanava nel tempo lo spettro del fallimento, iniziava un febbrile lavorio "creativo", quasi una gara, per individuare soluzioni possibili per reagire a un ricorso per il fallimento quando fosse trascorso il periodo di improcedibilità

Così, nel tentativo di contribuire a un dialogo costruttivo sono state proposte "ricette molto diversificate" nel tentativo di reagire all'evento (il Covid-19) che: 1) ha colpito improvvisamente tutte le imprese, indipendentemente dall'oggetto, dal settore, dalla localizzazione, e dal piano industriale in corso; 2) investe, in molti casi, imprese sane che a fine 
2019 non mostravano segni di crisi; 3) non è riconducibile a decisioni gestionali. Nella prima fase le riflessioni hanno avuto ad oggetto il presupposto oggettivo, vale a dire la nozione di insolvenza nel tentativo di sbarrare il campo, con una sua reimpostazione, alle dichiarazioni di fallimento.

Trascorso il periodo di improcedibilità, come è possibile arginare il fallimento?

Quello del fallimento è un presupposto oggettivo che si fonda su un dato fattuale, istantaneamente rilevato dal giudice: la difficoltà o l'incapacità irreversibile di far fronte regolarmente alle obbligazioni contratte. L'incapacità irreversibile pesa sull'impresa che è costretta ad assumere una decisione affinché un atteggiamento dilatorio non determini un aggravamento del dissesto.

Lo stato d'insolvenza, secondo la norma citata, si estrinseca in una situazione oggettiva e non transitoria di incapacità ad adempiere le obbligazioni a tempo debito e con mezzi normali.

Dalla valutazione dell'insolvenza esula qualsiasi esame sulla gestione passata dell'impresa e, quindi, sulla genesi della crisi. L'insolvenza si risolve in uno stato irreversibile di squilibrio patrimoniale e finanziario, nel quale è assente l'indagine sulle sue cause.

L'insolvenza, sia colpevole che incolpevole, attiene esclusivamente all'elemento patrimoniale e finanziario dell'impresa ed è pertanto a questi fattori che occorre fare riferimento per accertare la presenza del presupposto oggettivo che impone l'adozione dello strumento concorsuale. Una gestione corretta non esclude l'insolvenza, così come una gestione scorretta non può essere assimilata all'insolvenza al punto di determinare da sola l'apertura della procedura concorsuale.

Per il legislatore l'insolvenza si concretizza in una situazione d'impotenza economica, determinata dalla incapacità dell'imprenditore di far fronte regolarmente, e con mezzi normali, alle proprie obbligazioni, per il venir meno delle condizioni di liquidità e di credito. Ma da quali elementi potremo desumere la sussistenza di tale incapacità all'adempimento?

L'inadempimento al pari di altri atti o fatti, costituisce sintomo di possibile insolvenza, ma non vi si identifica. L'insolvenza poggia su diversi indici segnaletici, dei quali l'inadempimento non è neppure quello che meglio e più la svela.

Nella valutazione circa la sussistenza o meno dello stato d'insolvenza giuoca inoltre un ruolo importante la capacità o meno di reperire credito.

Il reperimento del credito costituisce il punto di forza nell'esclusione dell'insolvenza. Come più volte è stato affermato, anche una mancanza assoluta di liquidità non è fattore determinante per affermare l'esistenza dello stato d'insolvenza. 
L'assenza di liquidità può, infatti, costituire una fase momentanea dell'ente ove il piano industriale, la massa patrimoniale con la situazione creditoria da un lato, e l'esposizione debitoria dall'altra, possano esprimere ancora una certa dimensione concorrenziale dell'impresa.

Questa analisi della crisi indirizza, però, anche verso l'esame del credito. Gli interventi di terzi finanziatori debbono, infatti, essere analizzati perché saranno idonei ad escludere l'insolvenza solo se ripristino la regolarità degli adempimenti, costituendo un flusso di denaro fresco verso l'impresa, e non si pongano, invece, il limitato scopo di favorire un regolare ma momentaneo funzionamento del sistema, destinato, comunque, a subire la crisi della singola unità produttiva.

Se l'erogazione ha lo scopo di rifinanziare l'impresa saremo in presenza di una valutazione in positivo dell'impresa, di una fiducia che quell'iniziativa economica continua a trasmettere ai terzi. Il credito ottenuto sulla base della fiducia che l'impresa riscuote esclude l'insolvenza.

A queste considerazioni, sulle quali la giurisprudenza prevalente ha ripetutamente insistito e che ci appaiono ineccepibili là dove esse si soffermano sulla funzione del finanziamento rispetto all'impresa - se cioè quello si collochi nel contesto di un'attività in fieri oppure in un'attività che si sta esaurendo per fattori endemici -, possiamo aggiungerne altri che tengono conto del credito in relazione alla situazione patrimoniale.

Il credito, per escludere l'insolvenza, deve essere, infatti, proporzionato alla dimensione e alla capacità patrimoniale dell'impresa, costituendo, allora, uno strumento, sia pure indiretto e in tempi non brevi, per ampliarne la futura consistenza mediante un rafforzamento della dimensione concorrenziale dell'impresa. Se, invece, il credito ottenuto conduce solo ad un aumento dell'indebitamento senza aprire prospettive, neppure in tempi medio-lunghi, di risanamento, diviene fattore oltre che sterile, sintomatico di uno stato di crisi.

Questo del credito è un parametro che all'impresa "infetta dal Covid-19" non si può probabilmente applicare, trattandosi di un'attività che deve riposizionarsi sul mercato, per la quale una valutazione in termini di capacità creditizia potrebbe essere svolta soltanto sulla base della gestione trascorsa. Per queste imprese il credito può derivare soltanto sulla base di garanzie esterne, così come l'intervento legislativo emergenziale, se pur con molti chiaroscuri, rende possibile.

L'esame dell'art. 5 1. fall. deve poi soffermarsi anche sull'elemento della c.d. esteriorizzazione dello stato d'insolvenza quale condizione perché il giudice, sulla base di un'iniziativa ex art. 6 1. fall., entri nell'impresa. Autorevole dottrina osservava che "se fosse 
diversamente, se l'assoggettamento al fallimento fosse dovuto all'insolvenza in quanto tale, dovrebbe ammettersi la possibilità del suo diretto accertamento, mentre questo è sicuramente escluso, perché implicherebbe un'invasione nella sfera giuridica dell'imprenditore (per la necessità di una consulenza tecnica o comunque di un esame dei suoi affari), la quale invasione costituirebbe un attentato alla libertà individuale ed alla segretezza degli affari ed avrebbe poi ripercussioni inevitabili gravissime sul credito e si presterebbe infine ad abusi”.

La crisi d'impresa che il legislatore individua nell'insolvenza, quale rottura del rapporto creditorio, può essere originata da motivi i più diversi tra loro che non rilevano nell'indagine giudiziaria, perché la scelta dell'ordinamento di applicare la procedura liquidativa dissolutiva, per soddisfare i creditori e per liberare possibili risorse potenzialmente utili al mercato esclude l'esame delle cause.

Ciò non toglie che l'insolvenza, quale ultimo "atto" di numerose "sequenze", abbia una causalità varia che ha corroso, comunque, la vitalità dell'impresa per cui non può essere esperito alcun tentativo di risanamento, non essendo, ove l'impresa sia di normali-medie dimensioni, né utile, né conveniente per la collettività, in quanto generatore di ulteriori costi, superiori alle aspettative di soddisfazione.

Diversa fattispecie è quella che presenta l'impresa quando essa non sia momentaneamente in grado di adempiere con regolarità e con mezzi normali alle obbligazioni assunte, ma non possa attribuire tale stato a gravi carenze strutturali generatrici di una crisi economica.

La situazione dell'impresa vittima del lock-down ma sana anteriormente, potrebbe essere identificabile in una temporanea difficoltà, altrimenti oggi denominabile "insolvenza reversibile", come "quella che rende impossibile l'immediato e regolare soddisfacimento delle obbligazioni", che si differenzia dallo stato d'insolvenza, perché "vi deve essere la capacità oggettiva dell'impresa di riacquistare il suo normale equilibrio". Questa potrebbe essere la difesa di un imprenditore convocato ai sensi dell'art. 15 1.fall.

L'impossibilità temporanea è dovuta alla mancanza di capitali liquidi necessari agli ordinari pagamenti, conseguente alla sospensione delle attività produttive imposta dalle misure di contenimento del virus. Sarebbe questa una causa destinata, entro un determinato lasso di tempo, ad essere rimossa sulla base di una realistica analisi prognostica. L'irreversibilità dello stato d'insolvenza si oppone, così, alla reversibilità ed incidentalità della temporanea difficoltà. Quest'ultima può dar luogo, eventualmente, a singoli inadempimenti, a episodi, quindi, di obbligazioni insoddisfatte mentre l'insolvenza è uno stato che non si sostanzia solo in molteplici inadempimenti, perché se questi ne sono manifestazione, non esauriscono la fattispecie, né 
spesso ne costituiscono l'aspetto più rilevante. È la crisi economica che rende irreversibile l'impotenza a pagare.

Nel fallimento la composizione della crisi passa, così, attraverso la realizzazione della massa attiva e quindi la conseguente valutazione ha ad oggetto il patrimonio e la sua consistenza, mentre in una procedura preventiva al centro della valutazione è l'impresa quale organismo dinamico e non quale aggregato di beni aventi consistenza patrimoniale.

Diverso si mostra immediatamente il ruolo del presupposto oggettivo nelle due procedure. Lo stato d'insolvenza è anche misura organizzativa della procedura, mentre una temporanea difficoltà ricopre un ruolo circoscritto all'introduzione della procedura. Valutato lo stato del patrimonio, si esamina l'impresa perché il ripristino della solvibilità dovrà avvenire attraverso l'attività e non mediante cessioni di parti rilevanti e significative del complesso aziendale e, quindi, sarà reso possibile soltanto dalla continuazione dell'impresa sottoposta a controllo, secondo il modello organizzativo e dimensionale prescelto. A ciò deve aggiungersi che, dinanzi a una crisi di questa tipologia, l'impostazione delle tradizionali procedure concorsuali liquidative come strumento in primis per soddisfare i creditori e per riallocare $\mathrm{i}$ valori residui in altre iniziative economiche non appare funzionale alla realtà contingente che induce ad abbandonare il metodo della selezione darwiniana per un ausilio, ancorchè non indiscriminato, alle attività economicamente sane, affette da postumi da lock-down.

Si tratta di due diverse conformazioni della crisi del rapporto di credito: l'incapacità assoluta e insanabile, che può condurre solo alla liquidazione disgregatrice e la crisi finanziaria e transeunte, superabile continuando la gestione dell'impresa sulla base di un nuovo piano industriale e nel contempo programmando il soddisfacimento dei creditori sulla base di una proposta di ristrutturazione delle loro pretese.

A conclusione di questo esame dell'art. 5 1. fall. possiamo affermare che la norma non consente un'interpretazione evolutiva senza un intervento del legislatore che la disponga perchè l'impresa in stato di irreversibile impotenza a far fronte alle obbligazioni -e non più di difficoltà temporanea - ha dinanzi, come tappa obbligata, il percorso concorsuale.

\section{Alla ricerca del sistema migliore per fronteggiare la crisi da Covid-19: la scelta tra legge fallimentare e il codice della crisi e dell'insolvenza}

Appena iniziato il lock-down, prefigurandosi un ribaltamento negativo sulle imprese e immaginando fin da subito un esito concorsuale della vicenda, è balzata evidente la possibilità di scegliere tra il sistema concorsuale vigente, di cui è espressione la sedimentata e 
sperimentata Legge fallimentare, oggetto di plurimi interventi normativi e quello espresso nel Codice della crisi, testo normativo di grande dimensione, approntato con l'aspirazione (tradita però in corso d'opera) ad unificare normative sparse in distinte leggi e dotato di una gamma variegata di istituti, alcuni dei quali rivisitati mentre altri del tutto nuovi.

Privilegiando la stabilità non solo normativa ma anche applicativa, l'art. 5 del decreto Liquidità, procedendo alla modifica del comma 1, dell'art. 389 del CCII, prevede che tale Codice entri in vigore il $1^{\circ}$ settembre 2021, "salvo quanto previsto al comma 2", anziché, come avrebbe dovuto, il 15 agosto 2020, cioè nel pieno dell'emergenza economica derivante dalla diffusione del Covid-19.

Diverse le ragioni che hanno giustificato questa decisione.

Accanto al (lodevole) motivo "tecnico" di non voler testare le nuove procedure in un momento di crisi eccezionale, appesantendo il lavoro di giudici e professionisti - con gli inevitabili costi di adattamento per tutti i soggetti coinvolti -, si colloca quello, che è parso più urgente, di allontanare dalle imprese lo spettro degli strumenti d'allerta volti a provocare l'emersione anticipata della crisi per evitare che un eccessivo numero di imprese, vittime del lock-down, ricadesse in quel perimetro. Peraltro, il d.1. 2 marzo 2020, n. 9 aveva già differito al 15 febbraio 2021 l'entrata in vigore delle misure di allerta, così che il decreto "Cura Italia" riallinea i termini di entrata in vigore delle misure di allerta e del rimanente Codice della crisi.

È indiscutibile che "il sistema dell'allerta è stato concepito nell'ottica di un quadro economico stabile e caratterizzato da oscillazioni fisiologiche, all'interno del quale, quindi, la preponderanza delle imprese non sia colpita dalla crisi, e nel quale sia possibile conseguentemente concentrare gli strumenti predisposti dal codice sulle imprese che presentino criticità. In una situazione in cui l'intero tessuto economico mondiale risulta colpito da una gravissima forma di crisi, invece, gli indicatori non potrebbero svolgere alcun concreto ruolo selettivo, finendo di fatto per mancare quello che è il proprio obiettivo ed anzi generando effetti potenzialmente sfavorevoli”.

Il Codice della crisi, infatti, amplia la platea dei soggetti legittimati a chiedere la liquidazione giudiziale e intensifica i poteri del pubblico ministero. Saremmo stati, quindi, in procinto di avere una nuova disciplina delle crisi da sperimentare in uno dei momenti più delicati per le imprese e l'economia italiana dal dopoguerra ad oggi e che aiuterebbe le imprese, anziché ad uscire dalle "secche", a incamminarsi speditamente verso la liquidazione giudiziale.

È vero la filosofia di fondo del CCII privilegia la continuità delle imprese sane promuovendo a strumenti principe accordi e concordati peraltro muniti di misure protettive da condizioni e limiti temporali che sarebbero poco confacenti alla situazione attuale. Quando 
questi, però, non possano realizzarsi si impone la liquidazione giudiziale in quanto ci troviamo dinanzi a una disciplina che vuole emarginare il concordato liquidativo accusato di costituire un mezzo autogestito per uscire dal mercato.

Nel Codice della crisi l'una o l'altra soluzione (per conservare o per cessare) devono essere tempestive, se possibile immediate, tutto muovendo dall'accertamento e dalla qualificazione dello stato di crisi o d'insolvenza. Il giudice deve decidere sulla base di una fotografia non del film dell'impresa e, a meno che non sia dotato di una illuminata sensibilità, non accerta quel presupposto oggettivo sulla falsariga di "Sliding doors".

Mi pare, allora, - ecco l'argomentazione più convincente al rinvio dell'entrata in vigore di quel Testo legislativo - che le nuove disposizioni del CCII siano per lo più rivolte a facilitare la rapida soluzione delle crisi di impresa tramite la espulsione dal mercato delle imprese in difficoltà, piuttosto che attraverso una fase controllata di sospensione del giudizio sul loro stato (sul presupposto oggettivo) per consentire un rimodellamento del programma industriale in funzione del recupero.

Per questi motivi, ritengo, che non sia quello attuale il momento per soluzioni così "catartiche" come il CCII, più della attuale Legge fallimentare, propone.

Questa logica è sicuramente utile in un momento economico in cui la ripulitura del mercato porti a una circolazione della ricchezza e, quindi, a un ricambio. Oggi il ricambio è precluso dalla crisi generalizzata. Non dimentichiamoci, inoltre, che il nostro tessuto imprenditoriale è costituito in larga prevalenza da piccole medie imprese che sono molto più vulnerabili in questa circostanza e per le quali il ricambio è sempre scarsamente praticabile.

Sotto questi aspetti, per i casi in cui si debba o per volontà dell'imprenditore o perché la liquidazione sia ineludibile e indifferibile, è preferibile utilizzare la Legge fallimentare largamente sperimentata da giudici e professionisti, innovata e corretta nel corso degli ultimi quindici anni, anche se non pervasa dal respiro codicistico e dalle suggestioni provenienti dai recenti interventi Unionali.

Il legislatore "inseguro y urgido" - incalzato dagli eventi e in assenza di previsioni attendibili, - con la decisione di rinviare l'entrata in vigore del Codice della crisi ha sicuramente operato una scelta drastica, accantonando, per questa emergenza e fino al $1^{\circ}$ settembre 2021 , un testo legislativo pervaso da un'impostazione più moderna, in linea con le posizioni espresse in vademecum per le crisi dagli Organismi internazionali e per la cui redazione tanto impegno era stato profuso.

È comprensibile allora che dinanzi a questo ampio rinvio si siano levate voci o improntate a aperto e totale dissenso, o caratterizzate da ragionevoli dubbi circa un'opportunità 
mancata, vale a dire quella di far vivere immediatamente, inserendole nel vigente assetto ordinamentale della crisi, almeno quelle norme contenute nel CCII e utili nell'attuale fase economica. A questo proposito sono state fatte alcune segnalazioni di possibili "recuperi".

Alla ricerca di uno strumento stragiudiziale, ma "controllato", è stato fatto riferimento alla composizione assistita della crisi che costituisce un "tavolo negoziale", "un percorso preconcorsuale" gestito dall'OCRI e che può condurre alla stipulazione di un accordo depositato presso quest'organismo, pubblicabile presso il registro delle imprese su richiesta del debitore e con il consenso dei creditori interessati, conosciuto soltanto da quei creditori che vi hanno partecipato e che sono tenuti alla riservatezza, privo di attestazione sulla fattibilità, sottratto a qualsiasi controllo giudiziale, che genera effetti soltanto tra gli aderenti, coperto (su richiesta al giudice presentata dal debitore) dalle azioni esecutive e dalle eventuali successive azioni revocatorie (ordinarie e fallimentari), così come lo sono le operazioni poste in essere in esecuzione di un piano ex art. $56 \mathrm{CCII}$, e favorito dalla altrettanto possibile sospensione degli obblighi societari (art. 20, comma 4, CCII).

Credo che l'attenzione verso la composizione assistita sia in verità suggerita dalla preoccupazione di supportare l'impresa nel suo percorso di recupero con l'intervento di un Organismo super partes, con compiti di advisor e di facilitatore nelle trattative.

Il sentiero che conduce alla composizione assistita, però, potrà avere una copertura dalle azioni esecutive e cautelari con una durata iniziale che "non può essere superiore a tre mesi" e che "può essere prorogata anche più volte, su istanza del debitore, fino al termine massimo" di ulteriori tre mesi, ma solo "a condizione che siano stati compiuti progressi significativi nelle trattative tali da rendere probabile il raggiungimento dell'accordo", solo su richiesta del debitore una volta che egli abbia presentato l'istanza per la soluzione concordata e sarà concessa dopo la sua audizione davanti all'OCRI e soltanto se tali misure protettive siano necessarie per condurre a termine le trattative in corso.

Questo sentiero può costituire anche - e questo è un aspetto da non trascurare soprattutto in questa fase economica in cui si cerca di tenere le imprese lontane dal fallimento - un piano inclinato verso la procedura liquidativa.

In caso di esito negativo delle trattative, infatti, il debitore è invitato a presentare una domanda di accesso ad una delle procedure di regolazione della crisi (art. 21, $1^{\circ}$ comma CCI) che, vista la perdita di continuità causata dal Covid-19 e l'incertezza nella redazione dei piani, difficilmente avrebbero, così come sono regolate, esito positivo. Più probabilmente il sentiero, allora, condurrebbe al fallimento. 
Per questi motivi ritengo che la composizione assistita mal si adatterebbe "a crisi di sistema coinvolgenti, come quella attuale (da Covid-19), un numero molto elevato di imprese e per un arco di tempo misurabile in anni (e non in mesi)".

Sicuramente i passaggi del CCII che avrebbero meritato, invece, una più meditata riflessione da parte del Legislatore sono quelli che si incontrano nella rivisitata disciplina, densa di implicazioni sociali, del sovraindebitamento. Alcune norme innovative del CCII sarebbero oggi di sicura utilità.

Intanto nel Codice della crisi, all'art. 66, grazie a una lettura estensiva della nozione di "debitore", acquista rilevanza la famiglia, direi il gruppo familiare indebitato. La disposizione è frutto di una visione realistica della famiglia - la causa ha radici comuni e si riflette su tutti e i debiti dell'uno sono per lo più debiti contratti per tutti e, quindi, di tutti e della funzione della procedura per far fronte al sovraindebitamento che non potrebbe, nel caso di un nucleo familiare, risolvere la crisi se non venissero coinvolte più entrate future e disponibilità attuali. L'art. 66 CCII può, quindi, essere letto come viatico non solo per far funzionare molte di queste procedure, a vantaggio dei soggetti debitori, ma anche per garantire il soddisfacimento dei creditori.

La norma, per un verso recependo arresti giurisprudenziali nei quali si era riconosciuto unitarietà dal punto di vista procedimentale al sovraindebitamento familiare e per un altro sulla scia della disciplina introdotta per la crisi e l'insolvenza del gruppo di imprese tesa a offrire un quadro globale di un unico fenomeno economico quandanche più e diversi siano i soggetti giuridici, contempla due distinte fattispecie. Quando i soggetti sono conviventi o quando il sovraindebitamento ha un'origine comune può essere presentato un unico piano di risoluzione della crisi (però con masse attive e passive separate).

È imposto, però, l'accesso al concordato minore in presenza anche di un solo familiare che svolga attività di libera professione o d'impresa ed allora un piano unitario potrà essere presentato ma secondo le regole che governano il concordato. Il fatto che tale norma sia contenuta nella sezione relativa alle disposizioni generali ne determina comunque l'applicazione a tutte le procedure di sovraindebitamento e, quindi, anche al concordato minore.

Nel caso dell'art. 66 CCII, l'opportunità razionalizzatrice del piano della famiglia in essa racchiusa, potrebbe essere importata sia per il piano del consumatore che per l'accordo senza creare difficoltà processuali e contribuendo, invece, a facilitare la crisi da sovraindebitamento di molti nuclei. 
Infine, sarebbe utile anticipare - senza dubbio sarebbe il "recupero" più necessario l'esdebitazione del "sovraindebitato incapiente" (art. 283 CCII).

Il sottosistema per il sovraindebitamento si caratterizza per l'esdebitazione, ed oggi più di ieri, in quanto per il "debitore persona fisica" incapiente "oggi e domani" - perché neppure prospetticamente è ipotizzabile alcun guadagno che faccia apparire possibile l'applicazione di uno strumento concorsuale -, è stata introdotta anche la forma di esdebitazione "una tantum" che prescinde dal collegamento con una previa procedura liquidativa e, quindi, da un "qualche" soddisfacimento dei creditori.

Ratio sottesa alla disciplina in commento è di dare attuazione all'indicazione, proveniente dal legislatore europeo e recepita nella legge delega (1. 19 ottobre 2017, n. 155), di rendere più efficaci le procedure di insolvenza, di ristrutturazione e di liberazione dei debiti: efficacia che, con l'istituto regolamentato nell'art. 283, si rafforza, atteso che in tal caso viene offerto al debitore un beneficio avente carattere straordinario, i cui effetti si producono non già in esito ad una procedura concorsuale, ma al di fuori e a prescindere dalla stessa.

Inoltre, si tratta di una liberazione integrale "condizionata" in quanto la legge sancisce "l'obbligo di pagamento del debito entro quattro anni, laddove sopravvengano utilità̀ rilevanti che consentano il soddisfacimento dei creditori in misura non inferiore al dieci per cento".

In altri termini, l'effetto esdebitativo, nel caso di specie, opera non già quale (possibile) effetto di una procedura liquidatoria, ma come beneficio «direttamente ricollegato alle circostanze che il debitore sia un nullatenente» e che non sia ipotizzabile un mutamento almeno in un immediato futuro della sua situazione patrimoniale: condizioni che renderebbero superflua e inutile l'apertura di una procedura.

La concessione è nelle mani del giudice che deve formulare un giudizio di merito e di convenienza sulla concessione del beneficio valutando discrezionalmente la meritevolezza del debitore sulla base della relazione redatta dall'OCC e nella quale viene esaminato anche l'operato del soggetto finanziatore, nella concessione del finanziamento, in particolare rispetto alla considerazione o meno del merito creditizio del debitore.

In sostanza emerge, quindi, che questa volta la valutazione della meritevolezza requisito per accedere agli strumenti per comporre il sovraindebitamento - deve scaturire da un esame dei comportamenti non solo del debitore ma anche del creditore. A questo proposito la funzione debtor oriented di queste procedure è più che chiara: il debitore in questione è soggetto scarsamente informato e supportato ex ante - la presenza ex post degli OCC quando la crisi è in atto e deve cercarsi una soluzione vuole assolvere a una funzione di ausilio - per cui la concessione del credito deve essere valutata con particolare accuratezza da parte del 
contraente forte, dotato di un apparato di conoscenze e di strumenti idonei ad effettuare una scelta razionale. In tale contesto il comportamento del finanziatore è preso in considerazione come fattore causativo dell'indebitamento o del suo aggravarsi che si ritorce sul diritto di autotutela del creditore.

\section{I problemi del legislatore dell'emergenza}

Il Legislatore è stato incalzato per un verso dal momento patologico - occorreva vaccinare contro il pericolo di immediato fallimento - per un altro dalla consapevolezza che, nelle situazioni estreme devono essere coniate misure specifiche, straordinarie come è l'evento che si è abbattuto contro il mondo.

Ha preso così corpo un diritto emergenziale, al momento, contrassegnato da due fasi, alle quali tra alcuni mesi se ne aggiungerà una terza.

Nella prima fase si sono adottate provvedimenti per sospendere l'applicazione di norme che potrebbero affrettare l'ipotesi fallimentare, mentre nella seconda si mira a indicare l'adozione di strategie e di strumenti, anche "alternativi”, per sopperire alle crisi nell'esecuzione dei contratti. La terza vedrà il l'utilizzo di strumenti concorsuali o esistenti o di nuovo conio ma comunque emergenziali.

Su un punto i legislatori della prima fase hanno coinciso: l'impresa deve poter analizzare e quantificare il danno che ha subito in conseguenza del lock-down per decidere se potrà o meno riposizionarsi nella dimensione concorrenziale detenuta antecedentemente. Si profilava il rischio che le imprese, in continuità fino a quel momento, divenute incapaci in conseguenza del lock-down di far fronte regolarmente agli impegni assunti e, quindi, alla luce dell'art. 5 1.fall., da ritenere in stato d'insolvenza, fossero dichiarate fallite senza poter dare loro la possibilità di tentare un recupero della solvibilità. Accanto a imprese contagiate dal Virus Corona-19 ve ne sono altre, però, erano già indebitate (e probabilmente già in una crisi latente) la cui situazione è stata esasperata dal blocco dell'attività e dalla conseguente caduta dei ricavi. Anche per queste pareva utile concedere un momento di pausa.

Nell'affrontare i problemi sollevati dal lock-down per le imprese il Legislatore ha iniziato dalla verifica delle regole esistenti nell'ordinamento per vedere se quelle fossero funzionali alla gestione di questa crisi. Si dovevano assumere provvedimenti per operare, con strumenti di diversa natura, un intervento sull'economia. 
Se l'attività legislativa non è mai scevra di problematiche di ordine soggettivo e spaziotemporale, nell'elaborazione di misure idonee a fronteggiare la crisi da Covid-19, lo è, infatti, ancora meno vista la straordinarietà e l'assenza di precedenti normativi.

In queste circostanze del tutto eccezionali, come è stato segnalato, quattro sono i problemi fondamentali che il legislatore incontra:

A) Il problema dell'ambito di applicazione - vale a dire stabilire quali norme debbono introdursi (soltanto con finalità di moratoria per congelare la situazione o anche nuovi strumenti con i quali gestire rapidamente la crisi?) e a chi debbano applicarsi (indistintamente a tutte le imprese, a prescindere dalla loro dimensione e natura? E poi, a tutte quelle che oggi si dichiarino in crisi o soltanto a chi dimostri di essere stato "vittima" del lock-down?).

Norme dall'orizzonte ristretto possono essere di scarsa utilità così come norme troppo ampie possono produrre conseguenze imprevedibili. Nella corretta soluzione di questo problema risiede l'utilità di uno strumento eccezionale che, ad avviso di chi scrive, deve avere la capacità di offrire soluzioni chiare, rapide, efficienti ed economiche riducendo così anche $i$ costi applicativi.

B) Il problema dell'equilibrio tra gli interessi in giuoco - questo è il punto più complesso con il quale si deve fare i conti.

In effetti in una situazione di crisi generalizzata, il legislatore ha il compito di realizzare l'equilibrio più efficiente tra l'interesse dei debitori (la continuità) e l'interesse dei creditori (il pagamento dei crediti), perché in questo equilibrio riposa l'interesse generale. Se si potenzia troppo il primo, si corre il rischio che i creditori, a loro volta impossibilitati a far fronte agli impegni assunti, diventino insolventi. Se si potenzia eccessivamente l'interesse dei secondi, però, si possono "strozzare" imprese, sane ma colpite ora dalla crisi Covid-19, che potrebbero essere però redditizie a medio termine, ritornando ad adempiere regolarmente.

Ecco il problema in sintesi: né eliminare immediatamente il debitore che oggi, a causa del Coronavirus, è in crisi di liquidità e quindi non adempie, né convertire i creditori in debitori. Nel primo caso si distruggono posti di lavoro e nel secondo egualmente.

È questa la situazione che la stasi da Covid-19 genera: tutti sono, se pure in diversa misura, debitori. Ciò richiede che lo strumento sia equilibrato per non determinare il crollo dell'intero tessuto imprenditoriale. Il principio della distribuzione delle perdite tra i creditori dell'impresa in crisi deve essere regolato con estrema cautela.

C) Il problema temporale - vale a dire della durata di questi strumenti per l'emergenza. È impossibile stabilirlo con un qualche grado di certezza quando ancora non si conosce la durata delle misure sanitarie. Le ipotesi più ottimistiche parlano di un inizio del recupero a partire 
dall'ultimo trimestre del 2021, quelle più realistiche posticipano questo inizio fino a tutto il 2022.

Termini temporali ristretti condurrebbero a un alto tasso di mortalità economica, anche se nel contempo eviterebbero ritardi da parte di imprenditori troppo fiduciosi in una ripresa e eventuali abusi da quelli in mala fede. Di contro con un arco temporale applicativo più ampio (due anni, come qualche Paese sta facendo) si offre una più realistica possibilità di ripristinare la continuità delle imprese sane colpite dal lock-down.

D)Quale obbiettivo assumere: selezionare o supportare?

Lo sfondo al quale pare naturale fare immediato riferimento per iniziative volte a riparare le lacerazioni prodotte sulle imprese dal lockdown è quello dell'Ordinamento della crisi, deputato a intervenire come insieme di regole per risolvere il conflitto tra debitore incapace a far fronte regolarmente alle proprie obbligazioni e creditori che pretendono il soddisfacimento. Ł̀ questo un sistema articolato tra più strumenti compositivi per il superamento della crisi e il mantenimento dell'impresa e uno liquidativo che, ancorché recentemente arricchito di potenzialità conservative di valori, è funzionale a depurare il mercato da iniziative economiche non più remunerative.

La presenza di un'impresa sana in stato crisi o, addirittura, in stato d'insolvenza (superabile) costituisce il discrimine tra i primi e il secondo che, invece, suppone un'incapacità irreversibile - sussistente anche quando l'attivo patrimoniale, pur superando il passivo, non sia liquidabile in tempi brevi (come oggi accadrebbe) - a far fronte regolarmente alle obbligazioni in quanto per lo più generata da una disfunzionalità di natura economica.

Sulla scorta di queste prime notazioni appare già messa in discussione un'utile funzione dell'attuale assetto concorsuale per ripristinare la continuità nelle imprese che siano state colpite dal lock-down. Nell'odierna situazione di crisi generale potrebbe apparire, tuttavia, estremamente difficile, se non impossibile, distinguere tra le imprese che possono proseguire la propria attività e quelle che invece non possono. Il rischio sarebbe che, applicando i principi previsti dall'ordinamento e sopra ricordati, la maggior parte delle imprese fosse considerata in crisi o, ancor peggio, insolvente e quindi dovesse essere liquidata.

L'attuale condizione di molte imprese, sane fino a febbraio, ma adesso incapaci di onorare le obbligazioni assunte condurrebbe - assumendo l'ordinamento fallimentare come unico strumento regolatore - a una selezione darwiniana del tessuto produttivo per il fallimento di numerose attività, atteso che gli strumenti alternativi in certi casi non potrebbero essere utilizzati per il loro alto costo e in altri, per i tempi serrati imposti, non darebbero risultati positivi. Anzi aggiungerebbero danno a danno. 
Ciò che occorre allora è un programma straordinario per la protezione e il recupero delle attività produttive meritevoli di essere conservate. Dovrebbe trattarsi di un programma straordinario, tenendo tuttavia presente che le crisi straordinarie tendono ad essere cicliche, dando origine a problemi straordinari, per cui sarebbe forse necessario creare regimi riservati per essere prontamente applicati in queste situazioni che cogliendo impreparato il Legislatore, vengono affrontate con eccessivo ritardo.

È illuminante, al riguardo, la disposizione dell'art. 69 (2) (a) dell'Insolvenzordnung austriaco. Questa norma stabilisce espressamente di prorogare il termine per depositare la domanda di accertamento dell'insolvenza quando quest'ultima è attribuibile a un disastro naturale (nel cui ambito rientra la pandemia).

Inoltre, il programma, al quale sopra facevo riferimento, dovrebbe essere indipendente dalle procedure concorsuali, ancorché di ristrutturazione, le quali dovrebbero continuare ad essere disciplinate così come disegnate dal Legislatore della "normalità". Il programma straordinario per il recupero delle attività produttive colpite dal Coronavirus dovrebbe comunque contenere quelle previsioni, ormai appannaggio delle tradizionali procedure concorsuali conservative, tese a garantire il mantenimento dei contratti in corso e a rinegoziare le obbligazioni. D'altra parte, per rinforzare la funzione di supporto all'impresa (che implica il ripristino della liquidità), sarebbero importanti misure complementari, come la protezione da azioni esecutive, gli incentivi a nuovi finanziamenti, in particolare al finanziamento dei soci.

È vero che gli strumenti concorsuali esistenti contengono quasi tutte le misure fin qui riferite. Nell'attuale situazione di crisi straordinaria, le imprese però necessitano di misure automatiche e indipendentemente dal ricorso ad altri istituti, cioè, senza necessità di iniziare una procedura. Probabilmente per gestire questa crisi siamo costretti a cambiare modello e progettare un trattamento sistemico con un approccio standardizzato.

\section{Ripensare la negozialità}

All'imprenditore in crisi da Covid-19 deve essere riconosciuta la possibilità di scegliere il proprio futuro tra gettare la spugna immediatamente o tentare di uscire dalle "secche".

L'imprenditore ha diritto, infatti, - in quanto meglio di altri conosce qual era l'andamento dell'attività prima del blocco emergenziale - di compiere una scelta tra abbandonare o rilanciare.

Con tutte le incertezze della situazione che rende difficile la redazione di un piano, può, comunque, esplorare la soluzione compositiva, peraltro facilitata nella tempistica, prima di 
giungere al fallimento che, comunque, se utilmente condotto, può sempre consentire di intraprendere un concordato.

Se deve essere concessa l'opzione di valutare la possibilità di recuperare la concorrenzialità, consequenzialmente quella deve essere supportata, sul piano esecutivo, con la inertizzazione durante la fase emergenziale - e per una durata congrua - delle procedure esecutive e, sul piano degli incentivi con misure: 1) lavoristiche, 2) finanziarie, 3) fiscali, 4) concorsuali. Questi sono punti assolutamente essenziali nell'ordine in cui sono enumerati che debbono essere considerati (soprattutto) rispetto sia alla piccola che alla media impresa.

Se i finanziamenti consentiranno alla maggior parte dei consumatori e delle imprese di evitare il default, il sistema concorsuale fungerà da sostegno più efficace. L'obiettivo dovrebbe essere quello di compensare gli effetti del lock-down con un supporto globale alla liquidità, non di salvare le imprese che hanno avuto problemi prima della crisi e che necessitano o, se possibile, di una ristrutturazione più approfondita $\mathrm{o}$, altrimenti, di essere liquidate.

Tuttavia, questa misura, che è stata predisposta dal d.1. 23/2020 (conv. In L. 40/2020) per fornire prestiti a coloro che ne hanno bisogno, in condizioni che vorrebbero essere vantaggiose e per un periodo limitato (tempo che peraltro tutte le imprese sarà necessario per adattarsi alla nuova situazione) e garantiti dallo Stato, non sembra sufficiente perché:

- si tratta di contratti bancari che devono essere stipulati in presenza di condizioni molto limitative (ergo "l'aiuto" per risolvere il problema è solo un ritardo);

- non vengono concessi facilmente o rapidamente, richiedono anche informazioni e documentazione complesse e la non pregressa esposizione deve essere evidente; e

- non sono facilmente accessibili da parte di microimprese e PMI che generalmente non dispongono di un modello commerciale in grado di gestire con successo le richieste di finanziamento perchè non sono né preparati per il requisito dell'accesso credito né hanno la disponibilità di una consulenza professionale.

- quel finanziamento con la garanzia dello Stato finirà per incidere sul contribuente.

Se il finanziamento non raggiunge la finalità desiderata, cos'altro si dovrebbe fare? Se i primi tre interventi sono propedeutici e funzionali alla ripartenza, non meno però è il quarto, non potendosi immaginare una ripresa senza un meccanismo flessibile, economico, snello, autogestito, che permetta la ristrutturazione dei debiti mentre i contratti proseguono ed è sospesa 
sia la disciplina, che scatta in caso di consistenti perdite di capitale e che impone di ricapitalizzare o di liquidare.

In ogni caso deve trattarsi di uno strumento chiaro e accessibile senza dover provare stringenti requisiti e passare da complesse procedure.

Su alcune di queste misure sono state predisposte, sull'ultimo punto - la predisposizione di un procedimento agile e flessibile - è stato compiuto quel tentativo, contenuto nel comma 5bis della L. 40/2020 e sopra illustrato, che ha dato luogo alla creazione di uno strumento di incerta natura.

Le misure per far ripartire l'impresa non possono consistere in malcerti rimaneggiamenti dell'esistente disciplina, né essere limitate a qualche mese ma devono accompagnare l'impresa per un ragionevole periodo di tempo.

Anche in tempi di crisi estreme come l'attuale devono, infatti, aprirsi spazi per soluzioni meno burocratiche che consentano una rinegoziazione delle obbligazioni retta dai canoni di collaborazione e lealtà connessi al solidarismo contrattuale. Ciò tanto più quando si avvertono non solo disequilibri tra le parti in termini di conoscenza tecnica e informazione (come sempre avviene) ma soprattutto i segnali di una crisi generalizzata per cui ciascuna delle parti è allo stesso tempo debitore e creditore.

È sicuramente essenziale una moratoria delle procedure di esecuzione sospendendo inoltre norme di diritto societario e concorsuale. Ciò produce l'effetto di bloccare l'iniziativa dei creditori, garantendo una tregua ai debitori. Tuttavia, proprio in virtù di questa tregua, viene rimosso l'incentivo a negoziare la ristrutturazione del debito perché i creditori temono che l'emergenza possa durare indefinitamente e che le loro richieste diventino irrecuperabili. Le negoziazioni, quindi, saltano.

Per altro verso, si deve tenere in conto che un ricorso massiccio alle procedure formali di ristrutturazione potrebbe causare gravi danni. Innanzi tutto, vi è un problema di costi che rendono quelle procedure scarsamente fruibili in questa fase. Inoltre, in una crisi sistemica, se la maggioranza delle imprese presentasse procedure formali di insolvenza, la struttura giudiziaria non sarebbe in grado di rispondere efficacemente a causa dell'eccessiva accumulazione di casi.

C'è di più: l'uso di lunghi procedimenti legali introdurrebbe un altro importante fattore di distruzione del valore, aggiungendo un nuovo elemento negativo alla grave crisi in corso.

Nell'attuale "insolpandemia", la legge fallimentare che mira a studiare la crisi e i suoi meccanismi di coping, deve offrire alternative diverse atteso che quelle tradizionali (che 
richiedono tempi lunghi e alti costi), già critiche per i tempi "normali", sono indiscutibilmente insufficienti. La sensazione è diffusa e molte in questi mesi sono state le proposte "alternative".

Cosa occorre? Le parti devono in primo luogo poter negoziare al riparo dalle azioni esecutive; avere a disposizione, per un periodo di due anni, uno strumento negoziale "ibrido", - ritengo, infatti, imprescindibile un controllo giudiziale sui requisiti e sulla gestione dell'impresa - aperto ad un ampio spettro di azioni e tecniche per ristrutturare i debiti e riorganizzare l'attività; caratterizzato da una gestione snella e da costi ridotti e, quindi, potenzialmente più efficace degli strumenti disciplinati nella nostra legge vigente e anche dello strumento - icasticamente definito "ircocervo" per la sua non ben definita natura - introdotto nel comma 5-bis dell'art. 9 L. 40/2020 che oltretutto sconterebbe una forte criticità sul piano dell'accesso al credito, dovendo la posizione dell'impresa ammessa a preconcordato essere classificata "a incaglio".

È, quindi, necessario che non vi siano ostacoli ad operazioni di conversione dei debiti in capitale. Le operazioni previste nel piano devono avvenire facilmente, rapidamente e a basso costo, senza dover incorrere in formalità complesse e costose, e senza necessità di autorizzazioni da parte dell'autorità giudiziaria. Il controllo sulla gestione operato da un commissario eviterebbe la prosecuzione in presenza di atti pregiudizievoli per i creditori o del venir meno della convenienza a proseguire.

È conveniente considerare misure lineari, chiare e fondamentalmente semplici, che risultino più convenienti rispetto agli esistenti strumenti di negoziazione preventiva senza tradurli in una procedura formale, articolata in controlli, attestazioni e autorizzazioni (come il concordato preventivo e, in parte, come gli accordi di ristrutturazione).

Tali misure potrebbero prevedere un codice di condotta per le negoziazioni (come, ad esempio, i Principi redatti da Insol); l'adozione di mezzi alternativi per risolvere rapidamente ed efficacemente i conflitti; la utilizzazione di un foro neutrale (come quello delle Camere di Commercio) che possa mettere a disposizione mediatori e arbitri di provata esperienza.

Tutto questo potrebbe essere di grande utilità per le piccole e medie imprese.

Ciò non significa che in questa fase la legge fallimentare debba essere completamente messa da parte dovendo costituire lo strumentario applicabile sia quando l'impresa non è in crisi da Covid-19, sia quando l'esperimento di ristabilire la continuità perduta a causa del Covid-19 è fallito.

Infine, - è bene ricordarlo - la legge fallimentare deve costituire l'alternativa efficiente nel caso in cui le negoziazioni non procedano a un ritmo ragionevole e costituire anche una barriera contro le tattiche dilatorie dei creditori. 


\section{Conclusioni}

La collaborazione, pernio del modello negoziale, deve cementarsi. Questo modello, completamente stragiudiziale o con intervento limitato del giudice, può risultare molto più efficace del complesso e costoso modello concorsuale giudiziale.

Il modello negoziale non è, infatti, imperniato sulla contrapposizione tra debitore e creditori, ma piuttosto sulla ricerca congiunta di una risposta adeguata al problema che li affligge tutti, cercando di puntare alla conservazione della relazione economica. L'accordo si proietta oltre la soluzione del debito.

Compito di chi assiste le imprese dovrebbe essere quello di incoraggiare formule che consentano la ristrutturazione dell'impresa e/o la rinegoziazione delle obbligazioni, modellandole sulle effettive capacità di pagamento del debitore, e in ultima istanza, quando la liquidazione sia inevitabile, perseguire l'ottimizzazione del soddisfacimento dei creditori.

I tribunali, da parte loro, devono sollecitare le parti a mettere in atto strategie di cooperazione, sanzionando i comportamenti scorretti dei debitori o dei creditori che cerchino soltanto di lucrare egoisticamente dalla procedura concorsuale. In questo senso, il fallimento deve replicare il modello di negoziazione in buona fede nei casi in cui la dimensione delle attività o delle passività giustifichi una struttura più complessa per determinare le alternative disponibili.

Senza dubbio resta da vedere se le conseguenze della crisi richiederanno ulteriori adattamenti delle legislazioni concorsuali una volta che, terminata l'emergenza, le imprese pur riprendendo l'attività necessiteranno di un periodo più o meno lungo - direttamente proporzionale alla capacità di resilienza - per ritornare alla dimensione concorrenziale quo ante.

È certo che non dovremo assistere a un ritorno a leggi fotografia come quelle che apparvero a fine degli anni settanta tendenti a salvare determinate imprese, bensì a interventi dettati da una situazione determinata da una pandemia e, quindi, diretti a tutte le imprese, in particolare quelle dei settori non ritenuti essenziali che sono coinvolte e che meritano - ad esclusione di quelle che già presentassero dai loro bilanci un forte squilibrio economicofinanziario - un ausilio, salvo diversa scelta dell'imprenditore, per poter riprendere l'attività dopo questa battuta d'arresto. Senza dubbio c'è un'esigenza di controllo sulla loro gestione nel periodo in cui usufruiranno delle risorse pubbliche e delle moratorie.

Come controllare? Una procedura come il preconcordato o preaccordo? Madaus e Wessels propongono "A compromise solution could provide for a deferral under available restructuring or even insolvency laws by allowing entrepreneurs (and creditors) to file for the 
protection of such regimes while at the same time staying any proceedings for the duration of emergency measures. Such an approach would require the entrepreneur to externalise the need for a deferral while it would not immediately bind scarce resources of the courts. With the termination of emergency measures, the motion could be withdrawn if the business is solid again".

Certamente, la legge fallimentare non è destinata a morire, ma piuttosto a essere reindirizzata, a funzionare come un punto d'incontro in cui, come in altre orbite giurisdizionali, i meccanismi alternativi di risoluzione dei conflitti assumono maggiore importanza.

\section{Riferimenti}

A. A. N. ROUILLON. El primer pilar legal para enfrentar una pandemia de insolvencia empresaria, in La Ley, 9 de junio 2020.

A. ROJO. Reflexiones sobre el Derecho concursal de emergência. In https://www.blog.fder.uam.es/2020/05/11/reflexiones-sobre-el-derecho-concursal-deemergencia/.

B. IVERSON. Get in Line: Chapter 11 Restructuring in Crowded Bankruptcy Courts. Management Science, 64 (2018): 5370, leggibile in https://doi.org/10.1287/mnsc.2017.2808.

C. BASTASIN, L. BINI SMAGHI, M. MESSORI, S. MICOSSI, P. C. PADOAN, F. PASSACANTANDO, G. TONIOLO. La risposta italiana alla crisi sanitaria. Pensare oggi il futuro del Paese, LUISS, 27 marzo 2020.

C. SERRA. A função (alternativa) do RERE como programa extraordinário para o apoio e a reanimação de empresas. www.revistadedireitocomercial.com ,2020-04-28.

E. MARTIN, IMF. Warns Small- and Mid-Sized Business Bankruptcies May Triple, leggibile.www.Bloomberg.com, 16 luglio 2020.

F. CESARE. Rimedi alla crisi epidemiologica nelle procedure di sovraindebitamento (02.05.2020).

F. DE SANTIS. La giustizia concorsuale ai tempi della pandemia. Fallimento, 2020, 612 ss.

F. FERRARA JR. - A. BORGIOLI. Il fallimento5, Milano, 1995, 143.

F. SANTANGELI - A. FABBI. Il (giusto) differimento ecc., cit. Negli stessi termini, M. IRRERA - E. FREGONARA, La crisi d'impresa e la continuità aziendale ai tempi del Coronavirus, in www.ilcaso.it, 15 aprile 2020, 3 ss.

G. LIMITONE. La forza maggiore nel giudizio sull'insolvenza, in www.ilcaso.it. 
J.L. GOLDENBERG. Repensar el derecho de la crisis desde la crisis, leggibile. www.dictumabogados.com.

K. AYOTTE - D. A. SKEEL, Jr. Bankruptcy or Bailouts. Journal of Corporation Law, 35 (2010).

M. FABIANI. Prove di riflessione sistematiche per le crisi da Emergenza Covid-19.

Fallimento, 2020, 590.

M. FERRO. Le nuove improcedibilità dei fallimenti dopo la legge n. 40 del 2020. Quotidiano giuridico, 11 giugno 2020.

M. MONTELEONE. L'incidenza del diritto emergenziale sullo stato di crisi e di insolvenza: la dirompente "insolvenza reversibile". Crisi d'impresa ed emergenza sanitaria, a cura di Ambrosini-Pacchi, Bologna, 2020.

N. ABRIANI - P. RINALDI. Emergenza sanitaria e tutela proporzionata delle imprese: oltre la domanda "tricolore". www.ilcaso.it, 4 giugno 2020.

P. F. CENSONI. La gestione delle crisi sistemiche delle imprese da COVID-19 fra Legge fallimentare e Codice della crisi e dell'insolvenza: il ritorno dell'Amministrazione controllata? (04.06.2020).

R. RORDORF. Il codice della crisi e dell'insolvenza in tempi di pandemia. www.giustiziainsieme.it

R. RORDORF. Il codice della crisi e dell'insolvenza in tempi di pandemia. Magistratura e giustizia sociale, Bari, 2020.

S. AMBROSINI. L'improcedibilità delle istanze di fallimento: ratio legis, tassatività della deroga e corollari applicativi. www.ilcaso.it, 29 maggio 2020.

S. AMBROSINI. La rinuncia al concordato preventivo dopo la legge (n. 40/2020) di conversione del "decreto liquidità": nascita di un "ircocervo"?, in www.ilcaso.it, 10 giugno 2020.

S. LEUZZI. Le procedure da "sovraindebitamento": profili generali, In A. CRIVELLI - R. FONTANA - S. LEUZZI - A. NAPOLITANO - F. ROLFI, Il nuovo sovraindebitamento dopo il Codice della crisi d'impresa e dell'insolvenza, Bologna, 2019.

S. MADAUS - B. WESSELS. Rescue of Business in Insolvency Law, European Law Institute, Vienna, 2017.

S. PACCHI, Quale sistema e quale strumento per la "insolpandemia"?, In Crisi d'impresa ed emergenza sanitaria, a cura di Ambrosini-Pacchi, Bologna, 2020. 